\title{
碳化锆陶瓷复合材料的制备、显微组织与性能
}

\author{
王东，王玉金 \\ (哈尔滨工业大学 特种陶瓷研究所, 哈尔滨 150001)
}

摘 要: 碳化锆 $(\mathrm{ZrC})$ 陶瓷复合材料具有熔点高、密度低、耐烧蚀的优点, 在超硬、航天防热、新能源等领域应用前 景广阔。本文概述了 $\mathrm{ZrC}$ 金属陶瓷和复相陶瓷、纤维增强 $\mathrm{ZrC}$ 复合材料的制备方法。着重介绍了粉末烧结、先驱 体转化、反应浸渗等工艺的应用, 并讨论了不同制备工艺下复合材料显微组织的特点。在总结两类材料力学性能和 烧蚀性能的基础上, 分析了各自的影响因素, 并指出 $\mathrm{ZrC}$ 金属陶瓷和复相陶瓷韧性低, 纤维增强 $\mathrm{ZrC}$ 复合材料烧蚀 层易剥落的问题。最后总结展望了 $\mathrm{ZrC}$ 陶瓷复合材料相关研究的发展趋势。

关 键 词: $\mathrm{ZrC}$ 复合材料; 制备; 组织结构; 性能; 综述

中图分类号: TB332 文献标识码: A

\section{Processing, Microstructure and Properties of ZrC Ceramic Composites}

\author{
WANG Dong, WANG Yu-Jin \\ (Institute for Advanced Ceramics, Harbin Institute of Technology, Harbin 150001, China)
}

\begin{abstract}
ZrC}$ ceramic composites have been proposed for a variety of applications in super-hard, thermal protection and new energy fields due to their excellent properties, such as high melting point, low density and good ablation resistance. The preparation techniques for $\mathrm{ZrC}$ cements, composite ceramics and fiber reinforced $\mathrm{ZrC}$ composites are reviewed, including powder sintering, precursor infiltration and pyrolysis and reactive melt infiltration. The effects of processing on microstructure of $\mathrm{ZrC}$ composite are discussed. Mechanical properties and ablation properties of $\mathrm{ZrC}$ ceramic composites are summarized. The problems, low toughness and spalling of ablation layer, for $\mathrm{ZrC}$ cements, composite ceramics and fiber reinforced $\mathrm{ZrC}$ composites are proposed, respectively. Finally, the trend of researches on $\mathrm{ZrC}$ ceramic composites is also prospected.
\end{abstract}

Key words: $\mathrm{ZrC}$ ceramic composites; preparation; microstructure; performance; review

碳化锆 $(\mathrm{ZrC})$ 作为超高温陶瓷家族的一员，具有 高硬度 $(25.5 \mathrm{GPa})$ 、超高熔点 $\left(3400^{\circ} \mathrm{C}\right)$ 以及良好的化 学稳定性, 被广泛应用于磨料、刀具、超硬薄膜材 料和耐火材料; $\mathrm{ZrC}$ 的密度 $\left(6.56 \mathrm{~g} / \mathrm{cm}^{3}\right)$ 低, 在超高温 氧化环境中, $\mathrm{ZrC}$ 表面生成 $\mathrm{ZrO}_{2}$ 膜能够保护基体材 料不被进一步侵蚀, 是理想的航天防热材料 ${ }^{[1]} ; \mathrm{ZrC}$ 中子吸收截面小, 耐辐射性能好, 有望应用于第四
代核能系统 ${ }^{[2]}$ 。此外, $\mathrm{ZrC}$ 还具有良好的热导率和较 低的辐射损失, 成为新一代太阳能热电接收器的候 选材料 ${ }^{[3-4]}$ 。 $\mathrm{ZrC}$ 作为基体或增强相与难熔金属 $(\mathrm{W}$ 、 $\mathrm{Mo} 、 \mathrm{Nb}$ 等 $)^{[5-8]}$ 和高熔点化合物 ${ }^{[9-18]}$ 复合, 可以降低 烧结温度, 改善彼此性能, 得到轻质、高强韧、抗氧 化的 $\mathrm{ZrC}$ 金属陶瓷或复相陶瓷。利用连续(炭)纤维 增韧 $\mathrm{ZrC}$ 基体，可以有效克服 $\mathrm{ZrC}$ 陶瓷韧性差、可

收稿日期：2014-09-19; 收到修改稿日期：2014-11-23

基金项目：国家自然科学基金(51172052); 新世纪优秀人才支持计划(NCET-13-0177); 中央高校基本科研业务费专项资金 National Natural Science Foundation of China(51172052); Program for New Century Excellent Talents in University (NCET-13-0177); Fundamental Research Funds for the Central Universities

作者简介: 王 东(1986-), 男, 博士研究生. E-mail: wangdong_tz@163.com

通讯作者: 王玉金, 教授. E-mail: wangyuj@hit.edu.cn 
靠性低等不足, 获得可靠性高、抗热震、耐烧蚀的 炭纤维增强 $\mathrm{ZrC}$ 复合材料 $\left(\mathrm{C}_{\mathrm{f}} / \mathrm{ZrC}\right)^{[19]}$ 。本文将对这 两类材料的研究现状进行综述, 重点介绍粉末烧结 制备 $\mathrm{ZrC}$ 金属陶瓷、复相陶瓷，先驱体法和反应浸 渗法制备 $\mathrm{C}_{\mathrm{f}} / \mathrm{ZrC}$ 的工艺、材料的显微组织和性能, 最后对 $\mathrm{ZrC}$ 陶瓷复合材料相关研究做几点展望。

\section{1 制备工艺}

\section{1 $\mathrm{ZrC}$ 金属陶瓷和复相陶瓷的制备}

$\mathrm{ZrC}$ 金属陶瓷和复相陶瓷最主要的制备方法是 粉末烧结, 此外还有反应浸渗以及电弧熔炼-定向 凝固等 ${ }^{[9]}$, 本文着重介绍前两种方法。

无压烧结(Pressureless Sintering, PLS)是最基本 的烧结方式, 产品尺寸不受限制, 简单易行。 $\mathrm{ZrC}$ 共 价键强, 自扩散系数低 ${ }^{[20]}$, 而烧结温度过高会导致 晶粒快速长大, 降低材料性能。目前已很少采用超 高温无压烧结的技术。细化粉末、增加缺陷可以降 低烧结温度。ZrC、Si 和石墨粉经高能球磨后 2100 ${ }^{\circ} \mathrm{C}$ 烧结得到的 $\mathrm{ZrC}-\mathrm{SiC}$ 复合材料, 致密度可以达到 $96.7 \%{ }^{[21]}$ 。在 $\mathrm{ZrC}-\mathrm{ZrO}_{2}$ 体系中, $\mathrm{ZrO}_{2}$ 和 $\mathrm{ZrC}$ 反应会 形成含较多缺陷的化合物, 起到增强扩散、加速致 密化的效果 ${ }^{[22]}$ 。烧结过程中, 生成与粉体润湿良好 的晶间液相可以促进烧结。添加 $20 \mathrm{vol} \% \mathrm{MoSi}_{2}$ 生成 液相后, $\mathrm{ZrC}-\mathrm{HfC} 、 \mathrm{ZrC}-\mathrm{ZrB}_{2}$ 的致密度超过 $95 \%{ }^{[17]}$ 。 胡志毅 ${ }^{[23]}$ 从调控烧结制度出发, 采用无压两步法 获得了板状晶自增韧 $\mathrm{ZrC}-\mathrm{ZrB}_{2}$ 复合材料。

热压/热等静压烧结(Hot Pressing/Hot Isostatic Pressing, HP/HIP) 是陶瓷致密化的常用手段。HIP$\mathrm{ZrC}-\mathrm{Mo}$ 在 $1800^{\circ} \mathrm{C}$ 烧结 $1 \mathrm{~h}$, 致密度超过 $98 \%{ }^{[24]}$, 热压致密化温度比 PLS 低 $350^{\circ} \mathrm{C}^{[25]}$ 。热压过程中 一般会加入助剂, B、C、 $\mathrm{B}_{4} \mathrm{C}$ 等可以除去粉末表面 的氧化层, 增强烧结性 ${ }^{[26]} ; \mathrm{Al} 、 \mathrm{Si} 、 \mathrm{La}$ 等在热压过 程中形成与粉末润湿良好的液相促进烧结 ${ }^{[27-29]}$; 难熔碳化物和 $\mathrm{ZrC}$ 反应, 生成含较多缺陷的化合 物, 起到增强扩散、加速致密化的效果 ${ }^{[26]}$ 。非化 学计量 $\mathrm{ZrC}\left(\mathrm{ZrC}_{x}\right)$ 的屈服应力随 $\mathrm{C}$ 缺位量增加而降 低, 可以降低 $\mathrm{ZrC}$ 陶瓷复合材料的热压致密化温 度 $^{[27-28]}$ 。

反应热压烧结(Reactive Hot Pressing, RHP)与热 压烧结相比, 具有更多优点: 该工艺一步完成反应 和致密化, 无需烧结助剂, 烧结温度低; 可以通过 调整反应物配比调控材料的微观结构, 并且这些原 位生成相化学兼容性好, 分布均匀 ${ }^{[30]}$ 。 $\mathrm{ZrC}-\mathrm{ZrB}_{2}$ $(-\mathrm{SiC})$ 复合材料一般通过下列反应得到 ${ }^{[30]}$ :

$$
\begin{gathered}
x \mathrm{Zr}+y \mathrm{~B}_{4} \mathrm{C}+(3 y-x) \mathrm{Si} \rightarrow \\
2 y \mathrm{ZrB}_{2}+(x-2 y) \mathrm{ZrC}+(3 y-x) \mathrm{SiC}
\end{gathered}
$$

$800^{\circ} \mathrm{C}$ 时, $\mathrm{ZrB}_{2}$ 虽然比 $\mathrm{ZrC}$ 稳定, 但是 $\mathrm{ZrC}_{x}$ 先于 $\mathrm{ZrB}_{2}$ 生成; $\mathrm{SiC}$ 则要在 $1100^{\circ} \mathrm{CZr}$ 完全转化为 $\mathrm{ZrC}$ 和 $\mathrm{ZrB}_{2}$ 后, 才会由 $\mathrm{Si}$ 与 $\mathrm{ZrC} 、 \mathrm{~B}_{4} \mathrm{C}$ 反应生成 ${ }^{[30]}$ 。由于上述 反应强烈放热, 在一定的条件下 $\left(T_{\mathrm{ad}} \geqslant 2073{ }^{\circ} \mathrm{C}\right.$, $\Delta H^{\theta}{ }_{298 \mathrm{~K}} / C_{\mathrm{p} 298 \mathrm{~K}}>2273^{\circ} \mathrm{C}$ ) 会触发自蔓延高温反应(Selfpropagation High-temperature Synthesis, SHS)。不同 研究者对 SHS 的作用看法不一。Wu 等 ${ }^{[10]}$ 通过高能 球磨细化反应物, 并控制烧结工艺, 制得的 $\mathrm{ZrB}_{2}$ $\mathrm{ZrC}-\mathrm{SiC}$ 复合材料在 $1600^{\circ} \mathrm{C}$ 即达到 $97.3 \%$ 的致密度, 而不发生 SHS 时致密度只有 $89.9 \%$ 。Qu 等 ${ }^{[30]}$ 则认 为 SHS 会形成非平衡相, 进而导致低致密度。其研 究显示: 不发生 SHS 的烧结物致密度可达 $99.6 \%$ 。 两个研究的结果不同也许是烧结工艺和原料配比不 同造成的。从式(1)中可以看出, $\mathrm{SiC}$ 的生成量受到物 料配比的限制。在原料中添加 $\mathrm{SiC}$ 可以制得 $\mathrm{SiC}$ 体 积分数大于 $20 \%$ 的 $\mathrm{ZrB}_{2}-\mathrm{ZrC}-\mathrm{SiC}$ 复合材料, 但 $\mathrm{SiC}$ 的含量过多对致密化过程不利 ${ }^{[31]}$ 。偏离(1)式配比加 入过量 $\mathrm{Zr}$, 利用高温液相中 $\mathrm{ZrB}_{2}$ 择优生长的特性, 调控微观结构, 可以获得自增韧 $\mathrm{ZrB}_{2}-\mathrm{ZrC}-\mathrm{Zr}$ 复合 材料 ${ }^{[32]}$ 。

无压或者热压烧结 $\mathrm{ZrC}$ 陶瓷复合材料需要在 $2000^{\circ} \mathrm{C}$ 左右保温几十分钟到几个小时。保温时间的 延长会导致组织粗化, 并增加成本。放电等离子烧 结(Spark Plasma Sintering, SPS)作为一种新颖的烧 结方法越来越受到研究者们的重视, 它具有升温迅 速、烧结快、操作简便、可重复性强、安全可靠等 优点 ${ }^{[33]}$ 。如采用热压工艺 $\left(2000^{\circ} \mathrm{C}\right)$ 烧结 $\mathrm{ZrB}_{2}-\mathrm{ZrC}$ $\mathrm{SiC}$, 要获得 $98 \%$ 的致密度, 保温时间需要超过 $1 \mathrm{~h}$, 而 SPS 在 2 min 内即可实现 ${ }^{[34]}$ 。压力和保温温度是 SPS 的两个重要参数。 $\mathrm{ZrC}_{x}$ 在 $1950^{\circ} \mathrm{C}, 40 \mathrm{MPa}$ 烧结 时致密度低于 $92 \%, 100 \mathrm{MPa}$ 烧结致密度可超过 $96 \%{ }^{[35]}$ 。 Zr、C、O 化合物的致密度, 随着保温温度 的升高而增大。共晶成分的 $\mathrm{ZrC}-\mathrm{ZrB}_{2}$ 在 $50 \mathrm{MPa}$-SPS 时, $1400^{\circ} \mathrm{C}$ 即开始致密化, 一直延续到 $1800^{\circ} \mathrm{C}$ 。但是 高温致密化后期晶粒粗化严重并产生大量晶间孔隙, 且温度过高会导致物质挥发、致密度下降, 这些弊 端不容忽视 ${ }^{[29,36]}$ 。此外, SPS 制备的材料尺寸普遍较 小, 这给材料性能测试和应用带来不便 ${ }^{[37]}$ 。

反应浸渗法(Reactive Melt Infiltration, RMI), 是将金属(合金)熔体浸渗到事先制备好的多孔陶瓷 坏体中, 利用熔体和坏体的反应得到复合材料的一 种方法。该方法具有材料致密、快速近净成型的优 点。 $\mathrm{Zr}$ 熔体渗入 $\mathrm{B}_{4} \mathrm{C}$ 坏体中, 制备的 $\mathrm{ZrC}-\mathrm{ZrB}_{2}-\mathrm{Zr}$ 
复合材料致密度超过 $99 \%$ 。通过调节 $\mathrm{Zr}$ 的残余量可 以控制断裂韧性 ${ }^{[38]}$ 。将 $\mathrm{B}_{4} \mathrm{C}$ 掺入 $\mathrm{ZrB}_{2}$ 中制得复合 坏体, $1900^{\circ} \mathrm{C}$ 渗入 $\mathrm{Zr}$, 反应 $10 \mathrm{~min}$ 即可获得相应材 料 ${ }^{[39]}$ 。Dickerson 等 ${ }^{[40-41]}$ 在 $1200 \sim 1300^{\circ} \mathrm{C}$ 将 $\mathrm{Zr}_{2} \mathrm{Cu}$ 合 金熔渗进 WC 坏体, 制备了致密、形状复杂的 $\mathrm{ZrC} / \mathrm{W}$ 基火箭发动机喉衬。虽然可以利用反应产物 的体积大于反应物体积的原理, 通过 3D 打印等先 进技术精确控制坏体气孔率来减少产品中的熔体残 余, 但是熔体残余似乎难以避免 ${ }^{[42]}$ 。残余的熔体会 在一定程度上影响材料的高温性能。此外, 对反应 浸渗过程中生成的一些新型多元化合物 ${ }^{[43]}$ 的研究 尚不深入。

\section{2 纤维增强 $\operatorname{ZrC}$ 复合材料的制备}

浆料热压烧结法 (Slurry Infiltration and Hot Pressing, SIHP)难以制备形状复杂的三维预制体增 强 $\mathrm{ZrC}$ 复合材料, 且高温高压使纤维严重受损，现 在已经较少采用。化学气相浸渍(Chemical Vapor Infiltration, CVI)成本相对较高, 制备周期长, CVI$\mathrm{C}_{\mathrm{f}} / \mathrm{ZrC}$ 的报道极少 ${ }^{[44]}$ 。下面介绍目前研究比较广泛 的两种方法, 可以低成本制备具有复杂形状的三维 预制体增强 $\mathrm{C}_{\mathrm{f}} / \mathrm{ZrC}$ 。

先驱体/浆料浸渍热解法(Precursor Infiltration and Pyrolysis, PIP) 采用液相先驱体或者混合陶瓷 粉体的浆料浸渍纤维骨架, 然后在一定温度下热 解，获得纤维增强陶瓷基复合材料。该法具有近净 成型、基体纯度好、原位细晶组织均匀等优点 ${ }^{[45]}$ 。 难熔金属碳化物的先驱体(难熔金属有机聚合物)合 成工艺复杂且产率低、危险性大, 故一般采用替代 办法 ${ }^{[46]} \mathrm{ZrC}$ 可以通过水基(氧氯化锆水合物 $\left(\mathrm{ZrOCl}_{2}\right)$ 混合酚醛树脂等含碳物质)或者醇基 $\left(\mathrm{Zr}\left(\mathrm{OC}_{4} \mathrm{H}_{9}\right)_{4}\right.$ 的 二乙烯基苯的溶液)先驱体在 $1700^{\circ} \mathrm{C}$ 左右发生碳热 反应获得。其中，水基先驱体 “干燥-固化-交联” 的时间较长, 裂解产物疏松易碎, 制备效率低; 醇 基先驱体具有较合适的浓度、粘度和交联性质，应 用较广。考虑到陶瓷基体的产率，在先驱体中添加 一定量的 $\mathrm{ZrB}_{2} 、 \mathrm{ZrC}$ 等的细粉, 可以提高超高温组分 体积分数、缩短制备周期。在先驱体中掺入聚碳硅 烷(PCS)或 $\mathrm{SiC}$ 细粉, 可以引入 $\mathrm{SiC}^{[47]}$ 来提高材料的 抗氧化能力。

浆料的流动性对浸渍过程影响较大。如果浆料 流动性差, $\mathrm{ZrC}$ 微粒填充纤维束间的大孔隙并出现 架桥现象, 热解后材料中会出现较多的微裂纹和孔 隙, 严重影响力学性能 ${ }^{[48]}$ 。Zhao 等 ${ }^{[49]}$ 使用 $\mathrm{Zr}\left(\mathrm{OC}_{4} \mathrm{H}_{9}\right)_{4}$ 和二乙烯基苯作为 $\mathrm{ZrC}$ 的先驱体, 得到 了 $3 \mathrm{D} \mathrm{C} / \mathrm{ZrC}$ 复合材料, 但是需要经过 16 次的 “浸
渍-固化-热解” 循环, 且成品的孔隙率高达 34.3\%, 严重影响材料的使用。寻求浸渍性能优、产率高、 廉价的新型 $\mathrm{ZrC}$ 先驱体, 防止热解时纤维的损伤、 晶粒的粗化是进一步研究的关键。

RMI 是制备纤维增强陶瓷基复合材料的另一种 有效方法。Ultramet 公司的复杂结构纤维增强难熔 碳化物推力室即用该法生产 ${ }^{[50]}$ 。将 $\mathrm{Zr}$ (合金)熔体浸 渗到多孔 $\mathrm{C} / \mathrm{C}$ 坏体内, 可在短时间内获得致密度较 高的 $\mathrm{C}_{\mathrm{f}} / \mathrm{ZrC}$ 。 $\mathrm{RMI}$ 的影响因素众多, 可在很大的范 围内改变工艺参数, 获得具有不同性能的 $\mathrm{C}_{\mathrm{f}} / \mathrm{ZrC}$ 。 主要调控因素为多孔坏体的性质(成分、孔隙率)和 熔体成分。合理设计熔体成分，可以降低熔渗温度， 减少高温制备过程中纤维的损伤。近期, 中国学者 在这方面做了大量工作 ${ }^{[11-54]}$ 。用 $\mathrm{Zr}-\mathrm{Si}$ 和 $\mathrm{Zr}$-B 等共 晶成分的熔体代替纯 $\mathrm{Zr}$, 在降低工艺温度的同时引 入 $\mathrm{SiC} 、 \mathrm{ZrB}_{2}$ 等物质，可望提高材料的高温抗氧化 性 ${ }^{[55-56]}$ 。但是, 共晶成分点 $\mathrm{Si}$ 和 $\mathrm{B}$ 的含量非常少, 产品中 $\mathrm{SiC} 、 \mathrm{ZrB}_{2}$ 的含量不足以有效提高材料的抗 氧化性。Ultramet 技术中关于纤维保护层、多孔 $\mathrm{C} / \mathrm{C}$ 坏体的制备等关键部分尚未公开。未来，除需解决 以上技术难题外，浸渗过程中熔体与坏体的润湿、 浸渗行为、反应机制、反应动力学等基础问题也是 重要的研究方向。

\section{2 组织特征}

\section{$2.1 \mathrm{ZrC}$ 金属陶瓷和复相陶瓷的显微组织}

材料的微观结构取决于原料的性质(粒径、纯 度、形态等)和制备工艺。ZrC 陶瓷复合材料一般需 要在 $1600^{\circ} \mathrm{C}$ 以上烧结。高温容易使晶粒快速长大, 所以文献报道的晶粒尺寸一般大于 $1 \mu \mathrm{m}$ 。即使事先 将粉体破碎到亚微米量级, PLS/HP 后的晶粒尺寸也 大于 $1 \mu \mathrm{m}$ (图 1(a)), 如果将烧结温度提高 $200^{\circ} \mathrm{C}$, 晶 粒尺寸增大 4 倍(图 1(b) $)^{[21]}$ 。低温 RHP 可以获得 $\mathrm{ZrC}$ 细晶组织 $(<0.5 \mu \mathrm{m})$ (图 1(c) $)^{[31,57]}$; SPS 由于升温迅速, 保温时间短, 晶粒尺寸较小 (图 1(d) $)^{[34]}$ 。原料粉体中 含有 $\mathrm{ZrC}_{x}$ 或者与 $\mathrm{ZrC}$ 形成有限固溶体的碳化物 $(\mathrm{VC}$ 等)时, 晶粒会因为扩散加快容易粗化, 甚至在 1800 2000 ${ }^{\circ} \mathrm{C}$ 出现异常长大 $(d=50 \sim 100 \mu \mathrm{m})^{[58,26]}$, 造成 晶内气孔等缺陷(图 1(e))。 $\mathrm{B}_{4} \mathrm{C} 、 \mathrm{SiC}$ 等硬质碳化物助 剂颗粒分布在 $\mathrm{ZrC}$ 晶界上，能阻碍晶粒长大 ${ }^{[10,14]}$ 。

$\mathrm{ZrC}$ 晶粒长大时无择优取向，一般呈等轴状。 制备过程中形成较多对 $\mathrm{ZrC}$ 润湿良好的液相(如 Mo) 时, $\mathrm{ZrC}$ 晶粒球化(图 1(f) $)^{[59]}$ 。 $\mathrm{ZrB}_{2}$ 生长时具有择优 取向，据此，在 $\mathrm{ZrC}-\mathrm{ZrB}_{2} 、 \mathrm{ZrC}-\mathrm{ZrB}_{2}-\mathrm{SiC}$ 体系中可 

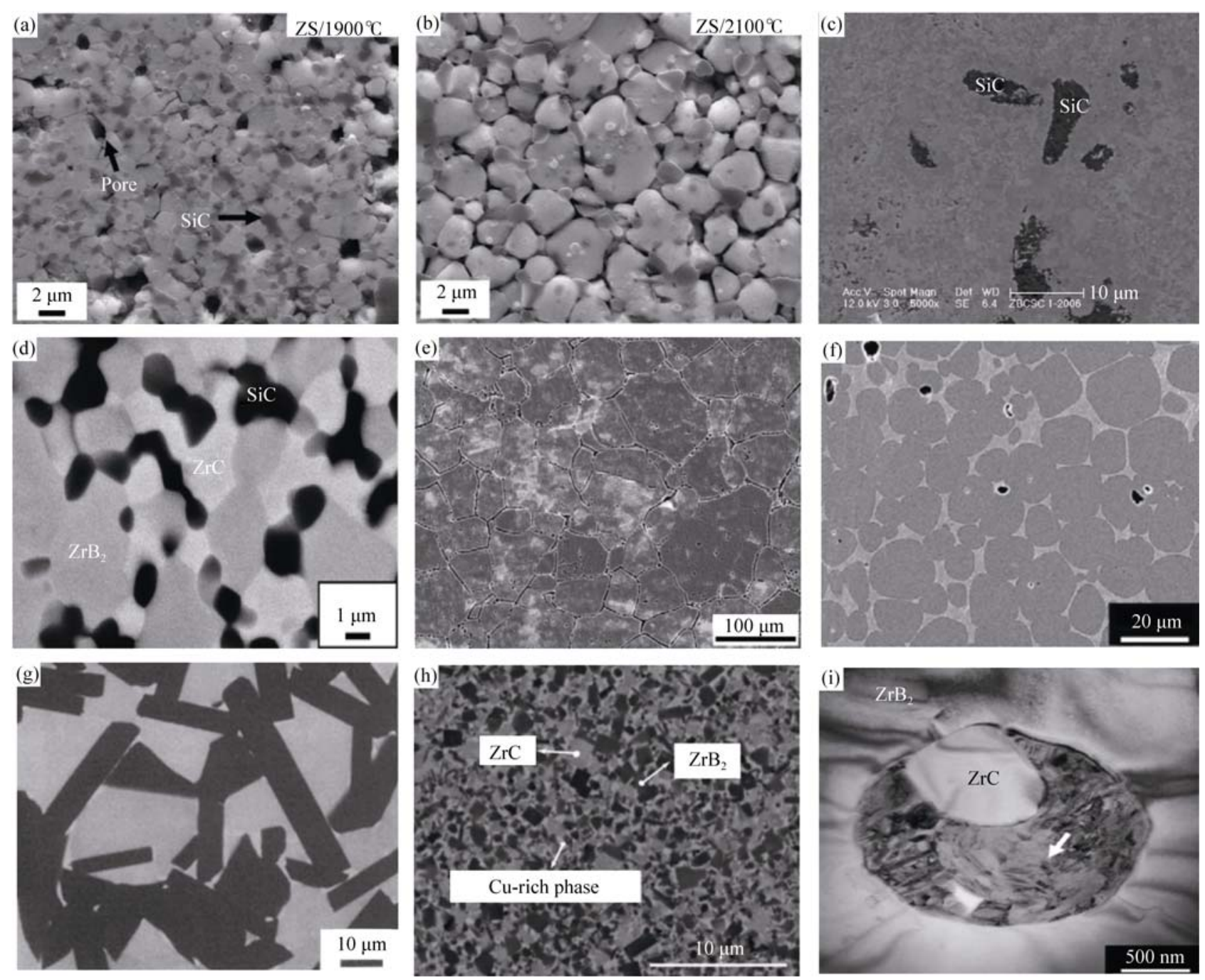

图 1 不同制备方法得到的 $\mathrm{ZrC}$ 金属陶瓷和复相陶瓷的形貌

Fig. 1 Morphologies of $\mathrm{ZrC}$ cements and composite ceramics prepared by different methods (a)PLS-ZrC-SiC at $1900^{\circ} \mathrm{C}$ using high-energy ball milling to enhance sinterability ${ }^{[21]}$; (b) PLS-ZrC-SiC at $2100^{\circ} \mathrm{C}$; (c)RHP-ZrC-ZrB ${ }_{2}-\mathrm{SiC}_{\text {fine }}$

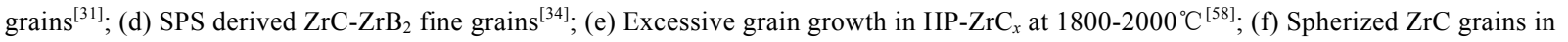
$\mathrm{ZrC}-\mathrm{Mo}^{[59]}$; (g) Backscattered electron image of $\mathrm{Zr}$ melt infiltrated into porous $\mathrm{B}_{4} \mathrm{C}$, white-remaining $\mathrm{Zr}$ gray-ZrC black-ZrB ${ }_{2}^{[60]}$; (h) Backscattered electron image of $\mathrm{Zr}_{2} \mathrm{Cu}$ melt infiltrated into porous $\mathrm{B}_{4} \mathrm{C}^{[61]}$; (i) Second phase containing $\mathrm{La}$ in $\mathrm{ZrC}_{\mathrm{ZrB}} \mathrm{Zr}_{2}$ composite ${ }^{[29]}$

以得到 $\mathrm{ZrB}_{2}$ 板状晶自增㓞结构。有研究表明: 高温 有利于 $\mathrm{ZrB}_{2}$ 板状晶发育, $1600^{\circ} \mathrm{C}$ 以下获得的 $\mathrm{ZrB}_{2}$ 板 状晶数量有限 ${ }^{[10,32]}$ 。类似的结果在 RMI-ZrC- $-\mathrm{ZrB}_{2}$ 中也有报道, 用纯 $\operatorname{Zr}\left(1900^{\circ} \mathrm{C}\right)$ 浸渗时, 生成了明显 的棒状 $\mathrm{ZrB}_{2}$ (图 $\left.1(\mathrm{~g})\right)^{[60]}$; 当改用 $\mathrm{Zr}_{2} \mathrm{Cu}\left(1200^{\circ} \mathrm{C}\right.$ ) 浸渗 时, 棒状 $\mathrm{ZrB}_{2}$ 的数量及长径比均下降 $(\text { 图 1(h) })^{[61]}$ 。

$\mathrm{Fe} 、 \mathrm{Ni}$ 等在 $\mathrm{ZrC}-\mathrm{ZrB}_{2}$ 晶间形成含其碳化物 的晶间相, $\mathrm{La}$ 除了可以存在于晶间和相间外, 还 能进入晶粒内部形成第二相粒子(图 1(i) $)^{[29]}$; 难 熔金属 $(\mathrm{Nb} 、 \mathrm{~W})$ 原子扩散进入 $\mathrm{ZrC}$ 晶格形成 $(\mathrm{Zr}$, $\mathrm{Me}) \mathrm{C}$ 固溶体, 界面处的互扩散则强化界面结合, 部分 $\mathrm{W}$ 原子在 $\mathrm{ZrC}$ 晶粒中析出 ${ }^{[6]} ; \mathrm{ZrB}_{2}$ 的(0001) 晶面和 $\mathrm{ZrC}$ 的(110)晶面匹配良好，二者形成较强 的键合 ${ }^{[9]}$ 。

\section{2 纤维增强 $\mathrm{ZrC}$ 复合材料的显微组织}

PIP- $\mathrm{C}_{\mathrm{f}} / \mathrm{ZrC}$ 的显微组织如图 2(a)所示, 可见基 体均匀分布到纤维束内部, 并存在较多的裂纹和孔 洞(气孔率达到 $34 \%$ )。先驱体交联裂解过程中体积 收缩, $\mathrm{ZrC}$ 基体呈海绵状分布在纤维束间, 且有明 显裂纹。 $\mathrm{ZrC}$ 基体呈海绵状, 先驱体交联裂解时, 溶剂挥发、裂解生成的小分子逸出和基体的收缩会 造成微米级小孔。后期浸渍过程中, 先驱体无法完 全填补孔洞，其自身收缩又会形成新的孔洞。由 EDS 结果可知, 纤维表面存在大量 $\mathrm{O}$ 和 $\mathrm{Zr}$ 元素, 说明中间产物 $\mathrm{ZrO}_{2}$ 与炭纤维之间存在界面反应和 界面扩散, 形成了 $\mathrm{Zr}_{x} \mathrm{O}_{y} \mathrm{C}_{z}$ 脆性化合物, 这可能使复 合材料性能降低 ${ }^{[46,49]}$ 。纤维以及基体中杂质第二相的 存在将限制晶粒的长大。例如, 在 $1600 \sim 1700^{\circ} \mathrm{C}$ 

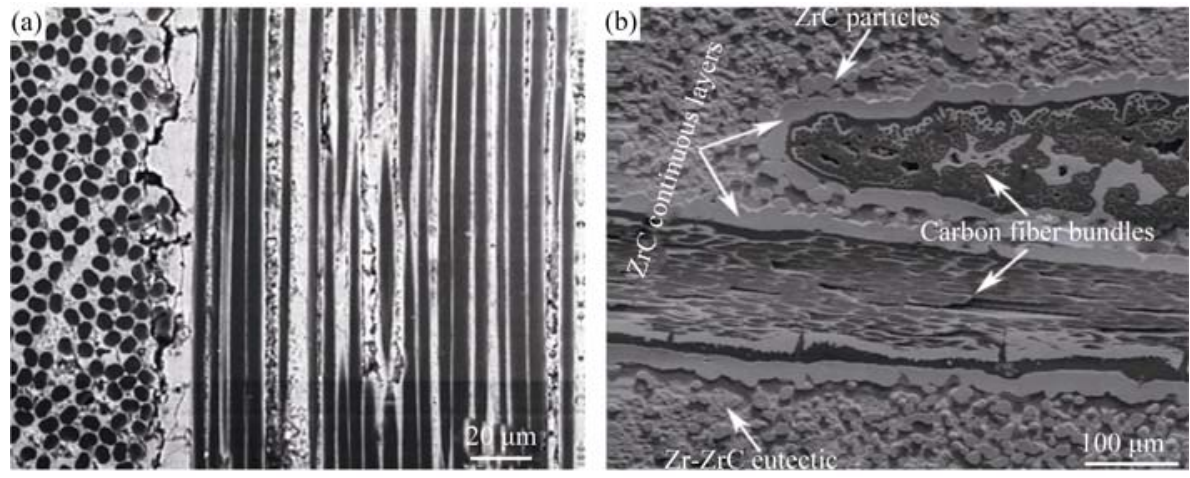

图 2 (a) PIP 法制备的 3D C $\mathrm{f} / \mathrm{ZrC}$ 横向/纵向显微组织 ${ }^{[49]}$ 和(b) RMI 法制备的 $\mathrm{C}_{\mathrm{f}} / \mathrm{ZrC}$ 复合材料的断面形貌 ${ }^{[66]}$

Fig. 2 (a) Horizontal and vertical microstructure of $3 \mathrm{D} \mathrm{C}_{\mathrm{f}} / \mathrm{ZrC}$ composite prepared by $\mathrm{PIP}^{[49]}$ and (b) section morphology of $\mathrm{C}_{\mathrm{f}} / \mathrm{ZrC}$ composite prepared by $\mathrm{RMI}^{[66]}$

热解先驱体得到的 $\mathrm{C}_{\mathrm{f}} / \mathrm{SiC}-\mathrm{ZrC}$ 复合材料中, $\mathrm{ZrC}$ 和 $\mathrm{SiC}$ 的晶粒尺寸只有 $25 \sim 40 \mathrm{~nm}^{[62]}$ 。对得到的材料进行热 处理, 发现 $\mathrm{SiC}$ 长成晶须, 覆盖在 $\mathrm{ZrC}$ 晶粒表面 ${ }^{[63]}$ 。

由 RMI 方法制备的 $\mathrm{C}_{\mathrm{f}} / \mathrm{ZrC}$ 复合材料与 PIP$\mathrm{C}_{\mathrm{f}} / \mathrm{ZrC}$ 存在明显差别。如图 2(b)所示, 复合材料致 密, 仅存在少量裂纹或孔洞 ${ }^{[64-65]}$ 。炭纤维束被致密 的 $\mathrm{ZrC}$ 层包围, 纤维束内部只分布少量基体。为了 防止材料及制备服役过程中的纤维损伤和退化，炭 纤维表面通过 CVD 沉积了 $\mathrm{ZrO}_{2} / \mathrm{HfN}(400 \sim 600 \mathrm{~nm})$ 界面层。基体为 $\mathrm{ZrC}$ 粒子呈 “岛状分布” 的 $\mathrm{ZrC} / \mathrm{Zr}$ 共晶组织, $\mathrm{ZrC}$ 颗粒内部有纳米相 $\alpha-Z r$ 。由于 $\mathrm{ZrC}$ 可 以形成 $\operatorname{ZrC}_{x}(x=0.5 \sim 1.0)$, 所以与熔渗 $\mathrm{Si}$ 生成 $\mathrm{SiC}$ 相 比, $\mathrm{Zr}$ 在复合材料中的残余量很小 ${ }^{[66]}{ }^{\circ} \mathrm{Zr}_{2} \mathrm{Cu}$ 熔渗多 孔 $\mathrm{C} / \mathrm{C}$ 时, 炭纤维束周围没有致密 $\mathrm{ZrC}$ 层, 基体主 要是呈八面体或切角八面体状的 $\mathrm{ZrC}$ 以及少量含 $\mathrm{Cu}$ 相 ${ }^{[67]}$ 。熔体换成 $\mathrm{Zr}-\mathrm{Si}_{8.8}$ 时, 基体是 $\mathrm{ZrC}-\mathrm{Zr}_{2} \mathrm{Si}$, 并 没有 $\mathrm{SiC}^{[68]}$ 。多元熔体熔渗的情况更加复杂, 这可 能是熔体各组元的碳化物形成能力以及反应机理的 差异造成的 ${ }^{[69]}$ 。

\section{3 性能}

\subsection{ZrC 金属陶瓷和复相陶瓷的力学性能}

表 1 给出了部分 $\mathrm{ZrC}$ 陶瓷复合材料的组织和力 学性能。由表 1 可以看出, 致密度对复合材料抗弯 强度的影响尤为明显。致密度 $99.6 \%$ 的 RHP-ZrB ${ }^{-}$ $\mathrm{ZrC}-\mathrm{SiC}$ 复合材料, 抗弯强度是致密度 $88.4 \%$ 材料的 2.5 倍 ${ }^{[30]}$ 。在致密度相近的条件下, 材料的强度服从 Hall-Petch 公式, 具有较小晶粒尺寸的材料将获得 较大的抗弯强度。 $\mathrm{ZrC}-40 \mathrm{vol} \% \mathrm{Mo}$ 中, $\mathrm{ZrC}$ 晶粒尺 寸从 $20 \mu \mathrm{m}$ 减小一个数量级, 材料的抗弯强度提 高 $70 \mathrm{MPa}^{[24-25]}$ 。类似的规律在 $\mathrm{ZrC}-\mathrm{ZrB}_{2}-\mathrm{Zr}$ 体系中
更加明显 ${ }^{[32,39]}$ 。

$\mathrm{HP}-\mathrm{ZrC}$ 的断裂韧性一般在2.0 3.0 $\mathrm{MPa} \cdot \mathrm{m}^{1 / 2[58]}$, 低韧性会极大限制 $\mathrm{ZrC}$ 复合材料的应用。Mo、Nb等 难熔金属在 $Z r C$ 晶间形成金属韧带，可以提高韧性。 HIP-ZrC-Mo的断裂韧性随Mo 含量增加, 由 $2.7 \mathrm{MPa} \cdot \mathrm{m}^{1 / 2}$ $(20 \mathrm{vol} \% \mathrm{Mo})$ 线性增大到 $6.6 \mathrm{MPa} \cdot \mathrm{m}^{1 / 2}(40 \mathrm{vol} \% \mathrm{Mo})^{[24]}$ 。 采用电弧熔炼方法制备的 $\mathrm{Mo}-40 \mathrm{~mol} \% \mathrm{ZrC}$ 的断裂韧 性达到了 $14.7 \mathrm{MPa} \cdot \mathrm{m}^{1 / 2[70]}$ 。 $\mathrm{Fe} 、 \mathrm{Ni}$ 等形成的晶间相 会使晶界脆化, La则可以形成晶间和晶内第二相增 㓞 $\mathrm{ZrC}^{[29]}$ 。复相陶瓷体系中 $\mathrm{ZrC}$ 含有 $40 \mathrm{wt} \% \mathrm{ZrO}_{2}$ 时, 断裂韧性可以提高到 $5.8 \mathrm{MPa} \cdot \mathrm{m}^{1 / 2}$, 但是增韧机制是 裂纹偏转还是 $\mathrm{ZrO}_{2}$ 的相变增韧尚不明确 ${ }^{[22]}$, 且 $\mathrm{ZrC}$ 晶格中固溶 $\mathrm{O}$ 后, 模量、硬度以及高温力学性能大幅 降低 ${ }^{[71]}$ 。理论和实践证明, 具有棒状或片状晶粒的 材料断裂韧性较高。 $\mathrm{Wu}$ 等 ${ }^{[10]}$ 用 RHP 制备的 ZrC$\mathrm{ZrB}_{2}-\mathrm{SiC}$ 复合材料, 在含板状 $\mathrm{ZrB}_{2}$ 时, 断裂韧性从 $2.56 \mathrm{MPa} \cdot \mathrm{m}^{1 / 2}$ 提高到 $5.2 \mathrm{MPa} \cdot \mathrm{m}^{1 / 2}$ 。Zhang等 ${ }^{[13]}$ 将其 进一步提高到 $7.28 \mathrm{MPa} \cdot \mathrm{m}^{1 / 2}$ 。当然, 增韧机制也包括 $\mathrm{SiC}$ 微粒使裂纹分叉偏转。Zhao 等 ${ }^{[72]}$ 发现, 一种含 $\mathrm{La} 、 \mathrm{Zr} 、 \mathrm{C} 、 \mathrm{~B}$ 的层状晶间相可以使 $\mathrm{ZrC}-\mathrm{ZrB}_{2}-\mathrm{SiC}$ 的 断裂韧性提高到7.9 MPa $\mathrm{m}^{1 / 2}$ 。目前看来, 自增韧是 复相陶瓷非常有效的一种韧化方式。

\section{2 纤维增强 $\mathrm{ZrC}$ 复合材料的力学性能和烧 蚀性能}

纤维增强 $\mathrm{ZrC}$ 复合材料的力学行为具有非线性 和尺寸效应，断裂时有明显的 “伪塑性” 行为，其强 度取决于基体(致密度、物相组成)、纤维骨架和界 面层。PIP-3D $\mathrm{C}_{\mathrm{f}} / \mathrm{ZrC}$ 的抗弯强度仅有 $107.6 \mathrm{MPa}^{[49]}$, 多次 PIP 循环有效提高致密度后, 材料的强度提高 一倍(表 2)。RMI- $\mathrm{C}_{\mathrm{f}} / \mathrm{ZrC}$ 致密度高, 室温强度优于同 等条件下 PIP 法制备的材料, 并且采用纯熔体反应 得到材料的强度好于采用复合熔体的材料强度。 
表 1 不同制备方法得到的 ZrC 金属陶瓷、复相陶瓷的组织和力学性能

Table 1 Microstructure and mechanical properties of ZrC composites prepared by different methods

\begin{tabular}{|c|c|c|c|c|c|c|}
\hline Composite & Method & $\begin{array}{c}\text { Relative } \\
\text { density } / \%\end{array}$ & $\begin{array}{c}\text { Grain } \\
\text { size } / \mu \mathrm{m}\end{array}$ & $\begin{array}{c}\text { Flexure } \\
\text { strength/MPa }\end{array}$ & $\begin{array}{l}\text { Fracture toughness } \\
\quad /\left(\mathrm{MPa} \cdot \mathrm{m}^{1 / 2}\right)\end{array}$ & Reference \\
\hline $\mathrm{ZrB}_{2}-\mathrm{ZrC}-\mathrm{SiC}$ & RHP & 88.4 & - & $204 \pm 5$ & $3.78 \pm 0.30$ & [30] \\
\hline $\mathrm{ZrB}_{2}-\mathrm{ZrC}-\mathrm{SiC}$ & RHP & 99.6 & - & $526 \pm 9$ & $6.70 \pm 0.20$ & [30] \\
\hline $\mathrm{ZrC}-\mathrm{Mo}$ & PLS & 97.8 & $\sim 20.0$ & 406 & - & [25] \\
\hline $\mathrm{ZrC}-\mathrm{Mo}$ & HIP & 98.7 & $2.2-5.5$ & 480 & 6.60 & [24] \\
\hline $\mathrm{ZrC}-\mathrm{ZrB}{ }_{2}-\mathrm{SiC}$ & RHP & 97.3 & $<2.0$ & $747 \pm 101$ & $5.2 \pm 0.4$ & {$[10]$} \\
\hline $\mathrm{ZrC}-\mathrm{ZrB}_{2}-\mathrm{SiC}$ & HP & 98.2 & $<5.0$ & $681 \pm 67$ & $5.65 \pm 0.16$ & [13] \\
\hline $\mathrm{ZrC}-\mathrm{ZrB}{ }_{2}-\mathrm{SiC}$ & RHP & 99.0 & $>5.0$ & $652 \pm 21$ & $7.28 \pm 0.32$ & [13] \\
\hline $\mathrm{ZrC}-\mathrm{MoSi}_{2}$ & SPS & 99.0 & 3.5 & $591 \pm 48$ & $3.3 \pm 0.4$ & {$[37]$} \\
\hline $\mathrm{ZrC}-\mathrm{ZrB}{ }_{2}-\mathrm{Zr}$ & RHP & 98.7 & 7.2 & 870 & 10.20 & {$[32]$} \\
\hline $\mathrm{ZrC}-\mathrm{ZrB} \mathrm{B}_{2}-\mathrm{Zr}$ & RMI & 99.5 & 15.0 & 570 & 11.50 & [39] \\
\hline
\end{tabular}

表 2 部分 PIP、RMI-C $/$ $/ \mathrm{ZrC}$ 的抗弯强度和断裂韧性

Table 2 Flexure strength and fracture toughness of PIP and RMI derived $\mathrm{C}_{\mathrm{f}} / \mathrm{ZrC}$

\begin{tabular}{ccccc}
\hline Composite & Method & $\begin{array}{c}\text { Flexure } \\
\text { strength/MPa }\end{array}$ & $\begin{array}{c}\text { Fracture toughness } \\
/\left(\mathrm{MPa} \cdot \mathrm{m}^{1 / 2}\right)\end{array}$ & Reference \\
\hline 3D C $\mathrm{f} / \mathrm{ZrC}$ & PIP(16cycles) & 107 & 7.03 & {$[49]$} \\
$3 \mathrm{D} \mathrm{C}_{\mathrm{f}} / \mathrm{ZrC}$ & PIP(20cycles) & 253.6 & 14.54 & {$[46]$} \\
$\mathrm{Felt}_{\mathrm{f}} / \mathrm{ZrC}$ & $\mathrm{RMI}$ & $120.4 \pm 2.8$ & $6.9 \pm 0.5$ & {$[73]$} \\
Felt C $_{\mathrm{f}} / \mathrm{ZrC}$ & $\mathrm{RMI}+1800^{\circ} \mathrm{CHT}$ & $104.7 \pm 3.2$ & $8.7 \pm 0.7$ & {$[73]$} \\
$3 \mathrm{D} \mathrm{C} \mathrm{f}_{\mathrm{f}} / \mathrm{ZrC}$ & RMI & $293.0 \pm 12.1$ & $9.8 \pm 0.9$ & {$[53]$} \\
\hline
\end{tabular}

$\mathrm{Zhu}$ 等 ${ }^{[73]}$ 真空高温热处理消除 $\mathrm{RMI}-\mathrm{C}_{\mathrm{f}} / \mathrm{ZrC}-\mathrm{Cu}$ 中的残 余合金，导致富 $\mathrm{Cu}$ 相挥发，造成气孔而使强度降低。 采用 $3 \mathrm{D}$ 编织的纤维预制体(纤维体积分数 $V_{\mathrm{f}}=$ (34.4 \pm 2.1$) \%$ )代替 $2.5 \mathrm{D}$ 炭毡预制体 $\left(V_{\mathrm{f}} \approx 30 \%\right)$, 在 $V_{f}$ 相 差不大的情况下, 复合材料强度提高近两倍。图 3 为 PIP-C $\mathrm{f} / \mathrm{SiC}-\mathrm{ZrC}$ 采用不同界面层材料时的 “弯曲强度位移” 曲线 ${ }^{[74]}$, 采用 $100 \mathrm{~nm}$ PyC 或 $200 \mathrm{~nm}$ PyC-SiC 复 合界面能够大大提高材料的强度, 且单一 $\mathrm{PyC}$ 界面效 果更优。调节界面层的厚度, 可以有效控制界面结合强 度。在 $\mathrm{RMI}-\mathrm{C}_{\mathrm{f}} / \mathrm{ZrC}$ 中, 不同厚度的 $\mathrm{SiC}$ 界面层对材料 力学性能影响较大。当 $\mathrm{SiC}$ 层厚度在 $0.05 \sim 0.5 \mu \mathrm{m}$ 之间 时; 复合材料的强度和没有界面层时相当, 当 $\mathrm{SiC}$ 层厚 度超过 $0.7 \mu \mathrm{m}$ 时, 复合材料强度下降明显 ${ }^{[75]}$ 。

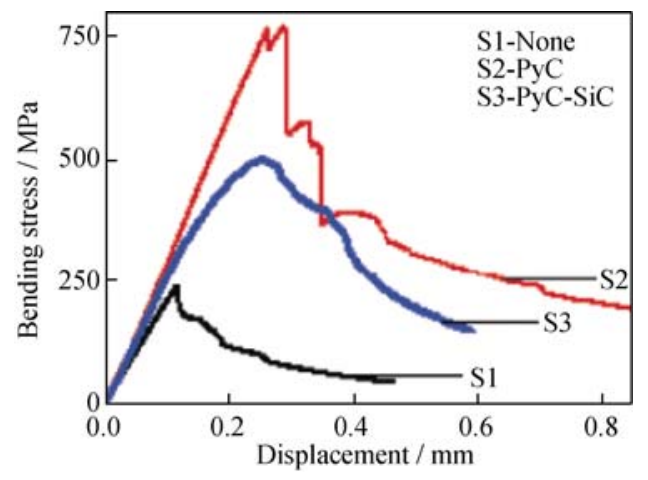

图 3 采用不同界面层材料时 $\mathrm{C}_{\mathrm{f}} / \mathrm{SiC}-\mathrm{ZrC}$ 的“弯曲强度-位移” 曲线 ${ }^{[74]}$

Fig. 3 Bending stress/displacement curves for composites with different interphases ${ }^{[7]}$
纤维增强 $\mathrm{ZrC}$ 复合材料除具有良好的抗热震性, 还具有优异的抗烧蚀性 ${ }^{[76]}$ 。材料优异的抗烧蚀性得益 于烧蚀产物 $\mathrm{ZrO}_{2}$ 较高的粘度、极低的蒸气压和氧扩散 率 ${ }^{[77]}$ 。当基体中含有少量易挥发相(如 $\mathrm{Cu}$ 及富 $\mathrm{Cu}$ 相) 时，这些物质可能起到 “发汗冷却” 的作用，减小线烧

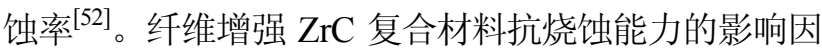
素主要有物相组成( $\mathrm{ZrC}$ 含量)、纤维骨架的编织方式和 材料的密度。图 4 给出了 $\mathrm{PIP}-\mathrm{C}_{\mathrm{f}} / \mathrm{ZrC}-\mathrm{SiC}$ 的烧蚀率随 $\mathrm{ZrC} / \mathrm{SiC}$ 含量比例的变化情况, 复合材料中 $\mathrm{ZrC}$ 含量越 高, 其抗烧蚀能力越强。 $\mathrm{ZrC}$ 烧蚀过程放出大量 $\mathrm{CO} / \mathrm{CO}_{2}$ 气体, 材料表面生成多孔 $\mathrm{ZrO}_{2}$, 这种缺少玻璃 相的烧蚀层粘附性差、易剥落 ${ }^{[78-79]}$ 。如何提高烧蚀层 对基体的粘附力, 是一项紧迫的任务。表 3 给出了

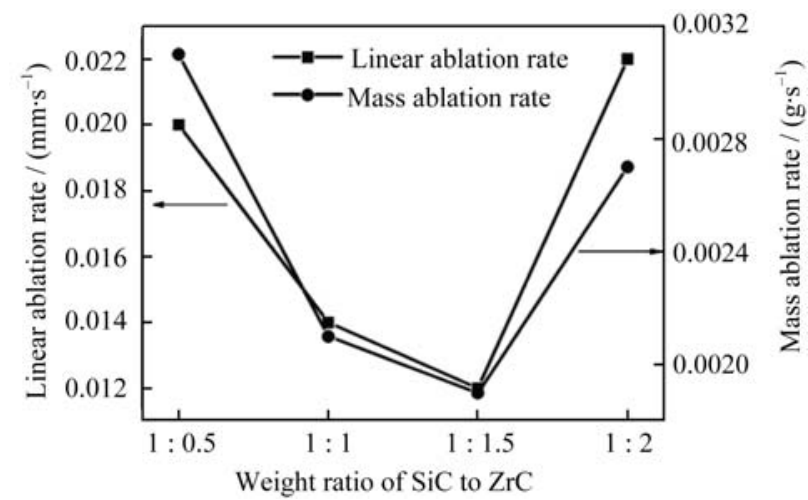

图 4 PIP- $\mathrm{C}_{\mathrm{f}} / \mathrm{ZrC}-\mathrm{SiC}$ 的烧蚀率随 $\mathrm{ZrC} / \mathrm{SiC}$ 含量比例的变化 ${ }^{[78]}$ Fig. 4 Ablation rates of PIP- $\mathrm{C}_{\mathrm{f}} / \mathrm{ZrC}-\mathrm{SiC}$ change with $\mathrm{ZrC} / \mathrm{SiC}$ $\operatorname{ratios}^{[78]}$ 
表 3 纤维编织方式及密度对 C/C-ZrC-TiC 烧蚀性能的影响 ${ }^{[80]}$

Table 3 Effects of fiber architecture and density on the ablation rates of C/C-ZrC-TiC

\begin{tabular}{ccccc}
\hline $\begin{array}{c}\text { Fiber } \\
\text { architecture }\end{array}$ & $\begin{array}{c}\text { Density / } \\
\left(\mathrm{g} \cdot \mathrm{cm}^{-3}\right)\end{array}$ & $\begin{array}{c}\text { Volume of } \\
\text { carbide } / \%\end{array}$ & $\begin{array}{c}\text { Linear ablation } \\
\mathrm{rate} /\left(\mathrm{m} \cdot \mathrm{s}^{-1}\right)\end{array}$ & $\begin{array}{c}\text { Mass ablation } \\
\mathrm{rate} /\left(\mathrm{mg} \cdot \mathrm{s}^{-1}\right)\end{array}$ \\
\hline FWPP & 2.50 & 18.71 & 1.96 & 2.28 \\
NIP & 2.50 & 18.70 & 1.80 & 2.10 \\
CWNP & 2.50 & 18.68 & -2.30 & 1.67 \\
CWNP & 2.38 & 16.33 & -0.25 & 1.92 \\
CWNP & 2.78 & 24.88 & -3.70 & 1.63 \\
\hline
\end{tabular}

FWPP -fine-weave pierced preform, NIP- needle-integrated preform, CWNP- chopped web needled preform

编织结构及密度对 RMI- C/C-ZrC- TiC 烧蚀性能的 影响。在密度相同时, 具有短切网状针刺毡结构的 复合材料的抗烧蚀性能优于细编穿刺毡和针刺整体 占; 当纤维骨架结构相同时, 材料密度越大, 抗烧 蚀性能越好。理论分析表明, 烧蚀产物 $\mathrm{ZrO}_{2} 、 \mathrm{TiO}_{2}$ 等熔化后在材料表面铺展的速度及其封闭烧蚀孔洞 的时间对材料线烧蚀率和质量烧蚀率起决定作用。 不同的编织方式导致封闭时间各不相同，进而影响 烧蚀率 ${ }^{[80]}$ 。

\section{4 总结与展望}

通过对国内外 $\mathrm{ZrC}$ 陶瓷复合材料研究现状的分 析，可以概括出以下几点:

1) $\mathrm{ZrC}$ 金属陶瓷、复相陶瓷以及纤维增强 $\mathrm{ZrC}$ 复合材料, 具有高硬轻质、高强韧性、耐烧蚀、抗 氧化的优点, 在超硬、航天防热、新能源领域具有 广阔的前景。

2) 粉末烧结是 $\mathrm{ZrC}$ 金属陶瓷、复相陶瓷主要制 备方法, 材料具有致密、强度高的特点。但是烧结 温度一般高于 $1600^{\circ} \mathrm{C}$, 如何降低工艺温度、减小成 本并更好地实现原位自生韧化将是烧结方法未来研 究的重点。先驱体转化和反应浸渗法是制备纤维增 强 $\mathrm{ZrC}$ 复合材料的主要方法, 可以实现近净成型。 先驱体法在组织均匀性上优势明显, 降低成本、提 高转化率是该方法亟待解决的问题; 反应浸渗法 能实现低成本快速致密化，如何控制反应、保护增 强体是该方法通向商业化道路上需要克服的两个 难题。

3) 粉末烧结已经能够实现 $\mathrm{ZrC}$ 金属陶瓷、复相 陶瓷致密、细晶、组织均匀的基本目标。先驱体法 制备的 $\mathrm{C}_{\mathrm{f}} / \mathrm{ZrC}$ 在组织均匀性上优势明显, 但是材料 致密度偏低。反应熔渗法则恰恰相反。结合两种方 法的优势, 可能获得具有更好组织结构、更低制备 成本的 $\mathrm{C}_{\mathrm{f}} / \mathrm{ZrC}$ 。

4) $\mathrm{ZrC}$ 金属陶瓷、复相陶瓷的强度已经很高，但
是偏低的断裂韧性会极大地限制它们的应用。如何 通过材料设计及组织调控, 克服其脆性和低可靠性 是未来研究的重要方向。通过纤维骨架、界面相的 设计，可以获得高强高韧、抗烧蚀性能优异的 $\mathrm{C}_{\mathrm{f}} / \mathrm{ZrC}$ 。如何提高烧蚀层对基体的粘附性，以期更好 的抗烧蚀效果, 有待进一步研究。

\section{参考文献:}

[1] JUSTIN J F, JANKOWIAK A. Ultra high temperature ceramics: densification, properties and thermal stability. The Onera Journal Aerospace Lab, 2011, 3: 1-11.

[2] KATOH Y, VASUDEVAMURTHY G, NOZAWA T, et al. Properties of zirconium carbide for nuclear fuel applications. Journal of Nuclear Materials, 2013, 441: 718-742.

[3] PIERRAT B, BALAT-PICHELIN M, SILVESTRONI L, et al. High temperature oxidation of $\mathrm{ZrC}-20 \% \mathrm{MoSi}_{2}$ in air for future solar receivers. Solar Energy Materials \& Solar Cells, 2011, 95: $2228-2237$.

[4] SANI E, MERCATELLI L, FRANCINI F, et al. Ultra-refractory ceramics for high-temperature solar absorbers. Scripta Materialia, 2011, 65: 775-778.

[5] ZHANG S C, HILMAS G E, FAHRENHOLTZ W G. Zirconium carbide-tungsten cermets prepared by in situ reaction sintering. Journal of the American Ceramic Society, 2007, 90(6): 1930-1933.

[6] SONG G M, WANG Y J, ZHOU Y. The mechanical and thermophysical properties of $\mathrm{ZrC} / \mathrm{W}$ composites at elevated temperature. Materials Science and Engineering A, 2002, 334: 223-232.

[7] LIDMAN W G, HAMJIAN H J. Reactions during sintering of a zirconium carbide-niobium cermet. Journal of the American $\mathrm{Ce}$ ramic Society, 1952, 35(9): 236-240.

[8] SCHOENMAN L. $4000^{\circ} \mathrm{F}$ materials for low-thrust rocket engines. Journal of propulsion and power, 1995, 11(6): 1261-1267.

[9] SORRELL C C, STUBICAN V S, BRADT R C. Mechanical properties of $\mathrm{ZrC}-\mathrm{ZrB}_{2}$ and $\mathrm{ZrC}-\mathrm{TiB}_{2}$ directionally solidified eutectics. Journal of the American Ceramic Society, 1986, 69(4): 
$317-321$.

[10] WU W W, ZHANG G J, KAN Y M, et al. Reactive hot pressing of $\mathrm{ZrB}_{2}-\mathrm{SiC}-\mathrm{ZrC}$ composites at $1600^{\circ} \mathrm{C}$. Journal of the American Ceramic Society, 2008, 91(8): 2501-2508.

[11] SILVESTRONI L, SCITI D, KLING J, et al. Sintering mechanisms of zirconium and hafnium carbides doped with $\mathrm{MoSi}_{2}$. Journal of the American Ceramic Society, 2009, 92(7): 1574-1579.

[12] SILVESTRONI L, SCITI D, BALAT-PICHELIN M, et al. Zirconium carbide doped with tantalum silicide: Microstructure, mechanical properties and high temperature oxidation. Materials Chemistry and Physics, 2013, 143: 407-415.

[13] ZHANG X H, QU Q, HAN J C, et al. Microstructural features and mechanical properties of $\mathrm{ZrB}_{2}-\mathrm{SiC}-\mathrm{ZrC}$ composites fabricated by hot pressing and reactive hot pressing. Scripta Materialia, 2008, 59: 753-756

[14] WU W W, ZHANG G J, KAN Y M, et al. Reactive hot pressing of $\mathrm{ZrB}_{2}-\mathrm{SiC}-\mathrm{ZrC}$ ultra high-temperature ceramics at $1800^{\circ} \mathrm{C}$. Journal of the American Ceramic Society, 2006, 89(9): 2967-2969.

[15] TSUCHIDA T, YAMAMOTO S. MA-SHS and SPS of $\mathrm{ZrB}_{2}-\mathrm{ZrC}$ composites. Solid State Ionics, 2004, 172: 215-216.

[16] YUNG D L, KOLLO L, HUSSAINOVA I, et al. Reactive sintering of ZrC-TiC composites. Key Engineering Materials, 2013, 527: 20-25.

[17] SILVESTRONI L, SCITI D. Microstructure and properties of pressureless sintered ZrC-based materials. Journal of Materials Research, 2008, 23(7): 1882-1889.

[18] HAN W B, WANG Z. Fabrication and oxidation behavior of a reaction derived graphite- $\mathrm{ZrC}$ composite for ultrahigh temperature applications. Materials Letters, 2009, 63: 2175-2177.

[19] 张立同 主编. 纤维增韧碳化硅陶瓷复合材料. 北京: 化学工业 出版社, 2009: 10, 78.

[20] BARNIER P, BRODHAG C, THEVENOT F. Hot-pressing kinetics of zirconium carbide. Journal of Materials Science, 1986, 21(7): $2547-2552$.

[21] ZHAO L, JIA D, DUAN X, et al. Pressureless sintering of ZrC-based ceramics by enhancing powder sinterability. International Journal of Refractory Metals and Hard Materials, 2011, 29: $516-521$

[22] MIN-HAGA E, SCOTT W D. Sintering and mechanical properties of $\mathrm{ZrC}-\mathrm{ZrO}_{2}$ composites. Journal of Materials Science, 1988, 23(8): $2865-2870$.

[23] 胡志毅. $\mathrm{ZrB}_{2}-\mathrm{ZrC}$ 复合材料的制备及力学性能研究. 哈尔滨: 哈尔滨工业大学硕士学位论文, 2012.

[24] LANDWEHR S E, HILMAS G E, FAHRENHOLTZ W G, et al. Microstructure and mechanical characterization of $\mathrm{ZrC}-\mathrm{Mo}$ cermets produced by hot isostatic pressing. Materials Science and
Engineering A, 2008, 497(1/2):79-86.

[25] LANDWEHR S E, HILMAS G E, FAHRENHOLTZ W G, et al. Thermal properties and thermal shock resistance of liquid phase sintered ZrC-Mo cermets. Materials Chemistry and Physics, 2009, 115: 690-695.

[26] WANG X G, LIU J X, KAN Y M, et al. Effect of solid solution formation on densification of hot-pressed $\mathrm{ZrC}$ ceramics with $\mathrm{MC}$ $(\mathrm{M}=\mathrm{V}, \mathrm{Nb}$, and $\mathrm{Ta})$ additions. Journal of the European Ceramic Society, 2012, 32: 1795-1802.

[27] WANG X G, ZHANG G J, ZHAO J, et al. High-strength ZrC ceramics doped with aluminum. Journal of the American Ceramic Society, 2014, 97(11): 3367-3370.

[28] WANG X G, ZHANG G J, XUE J X, et al. Reactive hot pressing of $\mathrm{ZrC}-\mathrm{SiC}$ ceramics at low temperature. Journal of the American Ceramic Society, 2013, 96(1): 32-36.

[29] KIM K H, SHIM K B. The effect of lanthanum on the fabrication of $\mathrm{ZrB}_{2}-\mathrm{ZrC}$ composites by spark plasma sintering. Materials Characterization, 2003, 50(1): 31-37.

[30] QU Q, HAN J C, HAN W B, et al. In situ synthesis mechanism and characterization of $\mathrm{ZrB}_{2}-\mathrm{ZrC}-\mathrm{SiC}$ ultra high-temperature ceramics. Materials Chemistry and Physics, 2008, 110(2/3): 216-221.

[31] RANGARAJ L, DIVAKAR C, JAYARAM V. Reactive hot pressing of $\mathrm{ZrB}_{2}-\mathrm{ZrC}_{x}$ ultra-high temperature ceramic composites with the addition of $\mathrm{SiC}$ particulate. Journal of the European Ceramic Society, 2010, 30(15): 3263-3266.

[32] GUO S, KAGAWA Y, NISHIMURA T. Tough hybrid ceramicbased material with high strength. Scripta Materialia, 2012, 67: 744-747.

[33] TOKITA M. Trends in advanced SPS spark plasma sintering systems and technology. Journal of the Society of Powder Technology Japan, 1993, 30(11): 790-804.

[34] GUO S Q, KAGAWA Y, TNISHIMURA T, et al. Mechanical and physical behavior of spark plasma sintered $\mathrm{ZrC}-\mathrm{ZrB}_{2}-\mathrm{SiC}$ composites. Journal of the European Ceramic Society, 2008, 28(6): $1279-1285$.

[35] GOUTIER F, TROLLIARD G, VALETTE S, et al. Role of impurities on the spark plasma sintering of $\mathrm{ZrC}_{x}-\mathrm{ZrB}_{2}$ composites. Journal of the European Ceramic Society, 2008, 28 (3): 671-678.

[36] GENDRE M, MAITRE A, TROLliARD G. A study of the densification mechanisms during spark plasma sintering of zirconium (oxy-)carbide powders. Acta Materialia, 2010, 58(7): 2598-2609.

[37] SCITI D, GUICCIARDI S, NYGREN M. Spark plasma sintering and mechanical behaviour of $\mathrm{ZrC}$-based composites. Scripta Materialia, 2008, 59(6): 638-641.

[38] JOHNSON W B, NAGELBERG A S, BREVA E. Kinetics of formation of a platelet-reinforced ceramic composite prepared by the 
directed reaction of zirconium with boron carbide. Journal of the American Ceramic Society, 1991, 74(9): 2093-2101.

[39] WOO S K, KIM C H. Fabrication and microstructural evaluation of $\mathrm{ZrB}_{2} / \mathrm{ZrC} / \mathrm{Zr}$ composites by liquid infiltration. Journal of Materials Science, 1994, 29(20): 5309-5315.

[40] DICKERSON M B, SNYDER R L, SANDHAGE K H. Dense near net-shaped, carbide/refractory metal composites at modest temperatures by the displacive compensation of porosity (DCP) method. Journal of the American Ceramic Society, 2002, 85(3): $730-732$.

[41] DICKERSON M B, WURM P J, SCHORR J R, et al. Near net-shape, ultra-high melting, recession-resistant $\mathrm{ZrC} / \mathrm{W}$-based rocket nozzle liners via the displacive compensation of porosity (DCP) method. Journal of Materials Science, 2004, 39(19): 6005-6015.

[42] LIPKE D W, ZHANG Y, LIU Y, et al. Near net-shape/netdimension $\mathrm{ZrC} / \mathrm{W}$-based composites with complex geometries via rapid prototyping and displacive compensation of porosity. Journal of the European Ceramic Society, 2010, 30(11): 2265-2277.

[43] ZHAO Y W, WANG Y J, ZHOU Y, et al. Ternary phase $\mathrm{Zr}_{x} \mathrm{Cu}_{y} \mathrm{C}_{z}$ in reactively infiltrated $\mathrm{ZrC} / \mathrm{W}$ composite. Journal of the American Ceramic Society, 2011, 94(10): 3178-3180.

[44] SAYIR A. Carbon fiber reinforced hafnium carbide composite. Journal of Materials Science, 2004, 39: 5995-6003.

[45] LOW I M, SAKKA Y, HU C F. MAX Phases and Ultra-high Temperature Ceramics for Extreme Environments. Hershey: Engineering Science Reference, 2013: 454.

[46] 赵丹. 耐超高温陶瓷先驱体及其复合材料的制备和性能研究. 长沙: 国防科学技术大学博士学位论文, 2011 .

[47] LI Q, DONG S, WANG Z, et al. Microstructures and mechanical properties of $3 \mathrm{D}$ 4-directional, $\mathrm{C}_{\mathrm{f}} / \mathrm{ZrC}$-SiC composites using $\mathrm{ZrC}$ precursor and polycarbosilane. Materials Science and Engineering $B, 2013$, 178(18): 1186-1190.

[48] 王其坤, 胡海峰, 陈朝辉. 先驱体转化法制备 $2 \mathrm{D} \mathrm{C} / \mathrm{SiC}-\mathrm{ZrC}$ 复 合材料中 $\mathrm{ZrC}$ 含量对材料结构性能影响研究. 航空材料学报, 2009, 29(4): 72-76.

[49] ZHAO D, ZHANG C R, HU H F, et al. Preparation and characterization of three-dimensional carbon fiber reinforced zirconium carbide composite by precursor infiltration and pyrolysis process. Ceramics International, 2011, 37(7): 2089-2093.

[50] Ceramics matrix composites.http://www.ultramet.com/ceramic matrix_composites.html[Eb/OL].2014-12-02.

[51] ZHU Y, WANG S, LI W, et al. Preparation of carbon fiber reinforced zirconium carbide matrix composites by reactive melt infiltration at relative low temperature. Scripta Materialia, 2012, 67(10): $822-825$

[52] ZHU Y, WANG S, CHEN H, et al. Fabrication of $\mathrm{C}_{\mathrm{f}} / \mathrm{ZrC}$ compos- ites by infiltrating $\mathrm{C}_{\mathrm{f}} / \mathrm{C}$ performs with $\mathrm{Zr}-\mathrm{Cu}$ alloys. Materials Letters, 2013, 108: 204-207.

[53] ZHU Y, WANG S, CHEN H, et al. Fabrication and characterization of 3-D $\mathrm{C}_{\mathrm{f}} / \mathrm{ZrC}$ composites by low-temperature liquid metal infiltration. Composites: Part B, 2014, 56: 756-761.

[54] CHEN S, ZHANG C, ZHANG Y et al. Influence of pyrocarbon amount in $\mathrm{C} / \mathrm{C}$ preform on the microstructure and properties of $\mathrm{C} / \mathrm{ZrC}$ composites prepared via reactive melt infiltration. Materials and Design, 2014, 58: 570-576.

[55] JIANG J, WANG S, LI W, et al. Preparation of 3D $\mathrm{C}_{\mathrm{f}} / \mathrm{ZrC}-\mathrm{SiC}$ composites by joint processes of PIP and RMI. Materials Science and Engineering: A, 2014, 607(23): 334-340.

[56] TONG Y, BAI S, YE Y, et al. Reactive melt infiltration of a $\mathrm{ZrB}_{2}$ modified $\mathrm{C} / \mathrm{ZrC}$ composite by a eutectic $\mathrm{Zr}-\mathrm{B}$ alloy. Materials Letters, 2015, 138(1): 208-211.

[57] RANGARAJ L, SURESHA S, DIVAKAR C, et al. Lowtemperature processing of $\mathrm{ZrB}_{2}-\mathrm{ZrC}$ composites by reactive hot pressing. Metallurgical and Materials Transactions A, 2008, 39A: 1496-1505.

[58] WANG X G, GUO W M, KAN Y M, et al. Densification behavior and properties of hot-pressed $\mathrm{ZrC}$ ceramics with $\mathrm{Zr}$ and graphite additives. Journal of the European Ceramic Society, 2011, 31:1103-1111.

[59] LANDWEHR S E, HILMAS G E, FAHRENHOLTZ W G. Processing of $\mathrm{ZrC}$-Mo cermets for high temperature applications, part II: pressureless sintering and mechanical properties. Journal of the American Ceramic Society, 2008, 91(3): 873-878.

[60] BREVAL E. Microstructure of platelet-reinforced ceramics prepared by the directed reaction of zirconium with boron carbide. Journal of the American Ceramic Society, 1992, 75(8): 2139-2145.

[61] ZHANG S M, WANG S, ZHU Y L, et al. Fabrication of $\mathrm{ZrB}_{2}-\mathrm{ZrC}$-based composites by reactive melt infiltration at relative low temperature. Scripta Materialia, 2011, 65(2): 139-142.

[62] PADMAVATHI N, KUMARI S, PRASAD V V B, et al. Processing of carbon-fiber reinforced $(\mathrm{SiC}+\mathrm{ZrC})$ mini-composites by soft-solution approach and their characterization. Ceramics International, 2009, 35(8): 3447-3454.

[63] LI H B, ZHANG L T, CHENG L F, et al. Fabrication of 2D $\mathrm{C} / \mathrm{ZrC}-\mathrm{SiC}$ composite and its structural evolution under hightemperature treatment up to $1800^{\circ} \mathrm{C}$. Ceramics International, 2009, 35(7): 2831-2836.

[64] ZOU L, WALI N, YANG J M, et al. Microstructural characterization of a $\mathrm{C}_{\mathrm{f}} / \mathrm{ZrC}$ composite manufactured by reactive melt infiltration. International Journal of Applied Ceramic Technology, 2011, 8(2): 329-341.

[65] NATALIE ALICE WALI. Fundamental Study of a Refrac- 
tory-based Carbon Fiber Reinforced Composite made by Reactive Melt Infiltration for Hypersonic Applications. Los Angeles: Doctoral Dissertation of University of California Los Angeles, 2011.

[66] ZOU L H, WALI N, YANG J M, et al. Microstructural development of a $\mathrm{C}_{\mathrm{f}} / \mathrm{ZrC}$ composite manufactured by reactive melt infiltration. Journal of the European Ceramic Society, 2010, 30(6): 1527-1535.

[67] WANG D, WANG Y, RAO J, et al. Influence of reactive melt infiltration parameters on microstructure and properties of low temperature derived $\mathrm{C}_{\mathrm{f}} / \mathrm{ZrC}$ composites. Materials Science and Engineering: $A, 2013, \mathbf{5 6 8 ( 1 5 )}:$ 25-32.

[68] TONG Y, BAI S, CHEN K. C/C-ZrC composite prepared by chemical vapor infiltration combined with alloyed reactive melt infiltration. Ceramics International, 2012, 38(7): 5723-5730.

[69] 张 鹏. 反应熔渗法制备 $\mathrm{C}_{\mathrm{f}} /(\mathrm{HfC}+\mathrm{MC})$ 复合材料机理及其性能 研究. 长沙: 国防科学技术大学硕士学位论文, 2011 .

[70] SUZUKI T, NOMURA N, YOSHIMI K, et al. 過共晶組織を有 する Mo-ZrC in-situ 複合材料の高温強度と室温勒性. [in Japanese] 日本金屬學會誌, 2000, 64(11): 1082-1088.

[71] GENDRE M, MAÎTRE A, TROLLIARD G. Synthesis of zirconium oxycarbide $\left(\mathrm{ZrC}_{x} \mathrm{O}_{y}\right)$ powders: Influence of stoichiometry on densification kinetics during spark plasma sintering and on mechanical properties. Journal of the European Ceramic Society, 2011, 31: 2377-2385.

[72] ZHAO L Y, JIA D C, WANG Y J, et al. ZrC-ZrB 2 matrix composites with enhanced toughness prepared by reactive hot pressing. Scripta Materialia, 2010, 63(8): 887-890.
[73] ZHU Y, WANG S, CHEN H, et al. Microstructure and mechanical properties of $\mathrm{C}_{\mathrm{f}} / \mathrm{ZrC}$ composites fabricated by reactive melt infiltration at relatively low temperature. Ceramics International, 2013, 39(8): 9085-9089.

[74] WANG Z, DONG S, ZHANG X, et al. Fabrication and properties of $\mathrm{C}_{\mathrm{f}} / \mathrm{SiC}-\mathrm{ZrC}$ composites. Journal of the American Ceramic Society, 2008, 91(10): 3434-3436.

[75] CHEN S, ZHANG Y, ZHANG C, et al. Effects of SiC interphase by chemical vapor deposition on the properties of $\mathrm{C} / \mathrm{ZrC}$ composite prepared via precursor infiltration and pyrolysis route. Materials and Design, 2013, 46: 497-502.

[76] ZHAO D, ZHANG C R, HU H F, et al. Ablation behavior and mechanism of $3 \mathrm{D} \mathrm{C} / \mathrm{ZrC}$ composite in oxyacetylene torch environment. Composites Science and Technology, 2011, 71(11): 1392-1396.

[77] http://www.ultramet.com/ceramic_protective_coatings.html\# refractory.

[78] FENG B, LI H, ZHANG Y, et al. Effect of $\mathrm{SiC} / \mathrm{ZrC}$ ratio on the mechanical and ablation properties of $\mathrm{C} / \mathrm{C}-\mathrm{SiC}-\mathrm{ZrC}$ composites. Corrosion Science, 2014, 82: 27-35.

[79] PAUL A, JAYASEELAN D D, VENUGOPAL S, et al. UHTC composites for hypersonic applications. American Ceramic Society Bulletin, 2012, 91(1): 22B-29B.

[80] ZENG Y, XIONG X, LI G, et al. Effect of fiber architecture and density on the ablation behavior of carbon/carbon composites modified by Zr-Ti-C. Carbon, 2013, 63: 92-100. 